\section{Freyre et \\ Maffesoli: \\ un lien}

\section{RESUMÉ}

Ce texte montre le lien théorique en ce qui concerne les aspects imaginaires des formes de vie entre l'auteur brésilien du début du siècle derniere, Gilberto Freyre, et le sociologue français, Michel Maffesoli, malgré les limites imposés par l'éloignèment dans l'espace et dans le temps entre les deux penseurs.

\section{RESUMO}

Este texto evidencia a ligação teórica relativa aos aspectos imaginários das formas de vida entre 0 autor brasileiro do início do século passado Gilberto Freyre e o sociólogo francês Michel Maffesoli, apesar da distância espaço/temporal entre os dois pensadores.

\section{PALAVRAS-CHAVE (MOT-CLÉS)}

- Sociologie (Sociologia)

- Michel Maffesoli

- Gilberto Freyre

\section{Muniz Sodré} UFRJ
GILbERTO FREYRE est peut-être l'auteur brésilien le plus proche de Michel Maffesoli. Il se peut que cette affirmation puisse avoir l'air d'un coup au minimum hasardeux, vu qu'il s'agit à la limite de rapprocher des engagements théoriques assez éloignés dans le temps et dans l'espace. Quand même, à l'encontre de tout ce qu'il est possible d'affirmer dans le cadre d'une intertextualité, l'analyse sociologique de Maffesoli se fait dans une perspective semblable à celle de Freyre, où le phenomène social est saisi dans une grande diversité d'aspects humains. On y a vraiment affaire à une visée théorique qui ne méconnaît pas les aspects imaginaires, sexuels, ethniques, éthiques, etc. des formes de vie.

Dans un compte-rendu sur Maîtres et esclaves, le chef-d'oeuvre de Freyre, Barthes a pu remarquer "un sens obsessionnel de la substance, de la matière palpable, de l'objet, si l'on veut, qui est au fond la qualité spécifique de tous les grands historiens ${ }^{1}$ Bien sûr, Freyre n'est pas proprement un historien : il est plutôt une hybridisation d'anthropologue, sociologue, historien et écrivain, dont la pluralité de méthodes fait analogie à son objet - le mélange ethnique du Portugais, de 1'Indien et du Nègre au Brésil. Cette sensibilité à l'Histoire totale permet qu'on le rapproche d'un historien comme Lucien Febvre, aussi bien que d'un sociologue comme Maffesoli, dont le goût pour l'hétérogène ou pour l'humoral dans les rapports de socialité est assez évident.

«L'humoral» dont il s'y agit en appelle à une attitude cognitive qui ne se passe pas de prêter attention aux phenomènes et aux faits menus concernant l'ethos, la manière d'être des individus et des collectivités, tout ce qui s'inscrit dans l'ici et maintenant de l'existence. Tout cela qui est exprimé au travers de figures comme le tragique, la théatralité, le rituel s'attache 
à une charge sensible, ainsi que le signale fort bien Maffesoli, que 1'on peut cerner en s'interrogeant sur le tressage d'une diversité de "manières " sociales, un monde sensible commun. On peut le nommer «socialité » ou Erlebnis, sí l'on déploit son sens $\mathrm{du}$ domaine individuel, d'après la tradition sociologique allemande, vers le collectif. Ce faire-valoir de la singularité constitutive du donné social, c'est un vrai point commun entre Maffesoli et Freyre.

C'est aussi vrai que la perspective axée sur la critique et la représentation classiques puisse remarquer c l'absence du element "lutte de classes » chez eux. Il y aurait quand même une virtuelle réponse commune à ce reproche : c'est important une visée sociologique qui retienne les modulations de la forme sociale, en suspendant méthodologiquement certaines catégories de la pensée prométhéenne tournée vers l'idéologie du progrès et de l'avenir. Il s'agit d'une mise en perspective des sujets qu'on pourrait aussi nommer "gaie »-- un " gai savoir », en bref. Chez Freyre, la gaieté ou la joie de penser garde des affinités avec la disposition viscérale et tragique chez certaines couches populaires du Brésil, dont les origines remontent aussi bien à la diaspora esclave africaine qu'à l'esprit « cordial » du pouvoir patrimonialiste brésilien.

Chez Mafesoli, il faut plutôt penser à Nietzsche, surtout quand celui-ci fait état d'une différence subtile entre la gaieté africaine e celle de 1’Europe à propos de la Carmen, de Bizet: "Cette musique est-elle gaie, mais non pas d'une gaieté française ou allemande. Sa gaieté est bien africaine. Le destin aveugle pése sur elle, son bonheur est bref, soudain, sans pitié» (L'Affaire Wagner). On y aperçoit une distinction forte entre deux types de régimes affectifs identifiés comme «gaieté » ou bien « joie». L'africain en est proprement tragique au sens nietzschéen du mot: "dire oui à la vie même dans ses problèmes les plus étranges et durs; la volonté de vivre se réjouit de son inépuisement au sacrifice où ses plus ele- vés répresentants sont immolés" -- tout ça j'appelle le dionysiaque, c'est bien ça dont j'ai eu l'intuition comme un pont que mène à la psychologie du poete tragique". ${ }^{2}$

Le sacrifice dont parle Nietzsche, c'est l'acceptation radicale de la communauté, le refus d'autopreservation physique ou morale de l'individu face aux intêrets majeurs du groupe. Le tragique, ce n'est pas la purge de la crainte ou de la compassion, tel qu'Aristote interprétait les oeuvres des grands poétes grecs, c'est plutôt l'expérience poétique du sacrifice qui conduit l'individu à être soi même dans un plaisir de transformation comprenant voire son propre anéantissement. Une joie tragique n'exclut pas le destin, puisqu'elle se produit au délà de la conscience, dans un débordement de forces, sans dépendances envers un passé ou un avenir, dans l'ici et maintenant d'une situation existentiellement excessive.

À l'esprit subtil, la dimension psychologique ne s'écarte pas de l'éthique, voire de l'esthétique quand il s'agit de bien cerner la joie. C'est le bon chemin pour arriver à la réfléxion de Nietzsche sur la joie tragique, aussi bien que pour lui faire une approche aux marges du cercle réfléxif de la philosophie occidentale, où la pensée sur le bonheur ou bien sur la béatitude comporte des analogies avec bien d'autres traditions réfléxives, à l'instar de celles d'Afrique ou de l'Inde.

Par le truchement de repères théoriques tels que «joie», "destin », «tragique », «éthique», «dyonisiaque » et " analogie », nous tenons à indiquer les pistes pour un rapprochement entre l'oeuvre de Maffesoli et lesdites «marges » du cercle réfléxif, où se sont toujours cantonnés ceux qui parient sur le relativisme de 1'Histoire occidental et son linéarisme finaliste, en faisant attention à l'experience du présent et à la vie au jour le jour. Nous sommes d'avis que l'émergence actuelle d'une nouvelle Cité humaine nos impose -- non seulement sur un plan intellectuel (le philosophique, qui entreprend une conceptualisation et 
une substantialisation de la différence), mais aussi sur les plans territorial et affectif -- d'écourter les distances qui séparent les différences telles qu'elles résultent du contentieux entre l'Histoire et le Destin, la raison et la passion, le logos et le pathos.

La puissance du sensible - de l'émotion-nel, du sentimental, du mythique, de l'affectif, - est sousjacente aux formes émergeantes de socialité et bien souvent en désaccord d'avec les institutions reconnues ou consacrées par le pouvoir de l'Etat, aussi bien que d'avec les grandes catégories de la rationalité censée d'être l'explication totale du monde. Ce faisant attention à la puissance du sensible, c'est le grand aspect novateur de l'oeuvre de Freyre à son époque. Toutes les proportions gardées, les contenus d'analyse mis à part, l'oeuvre de Maffesoli va à la rencontre de cette joyeuse attitude descriptive du social •

\section{Notas}

1 Barthes, Roland. Oeuvres Completes, tome I. Paris, Seuil, 1993, p. 211.

2 Nietzsche, F. 0 Crepúsculo dos Ídolos. p 143. 